\title{
Parametric excitation of Alfvén and acoustic waves
}

\author{
By NGUYEN THE HUNG \\ Centre de Recherches en Physique des Plasmas, \\ Ecole Polytechnique Fédérale de Lausanne, Switzerland
}

(Received 17 December 1973)

The nonlinear interaction between two Alfvén waves and a sound wave is studied, using the normal-mode approach. This leads, in a simple way to a set of coupled equations, and consequently to a dispersion relation for the waves under consideration. It is shown that a large-amplitude Alfvén wave can give rise to two distinct types of parametric instabilities, namely the oscillating and the purely growing waves. In each case, the expressions for the threshold pump intensity, the frequency shift and the growth rate of the excited waves are obtained. In particular, the results for a propagating pump under perfect frequency matching conditions are compared with those of Sagdeev \& Galeev.

\section{Introduction}

It is well known that an electromagnetic wave, above a certain threshold intensity, can excite other plasma waves through the parametric coupling mechanism. These effects, presumably responsible for various observed phenomena (e.g. anomalous reflection and enhanced absorption of laser radiation), have recently been of interest, both in the domain of laser fusion and in ionosphere research (DuBois, 1972).

In this paper we study the interaction of two Alfvén waves and a sound wave, using the coupled-mode theory, where both the frequency mismatch and the finite wavelengths of the waves are taken into account. One of the interests of this problem lies in the fact that a large amplitude Alfvén wave is an exact solution of the nonlinear MHD equations. Resonant harmonic generation being absent, the main nonlinear mechanism responsible for the decay of this wave should be its coupling with another Alfvén and a sound wave. This coupling process can be studied by various well-known methods (Kadomtsev 1965; Sagdeev \& Galeev 1969). Here, the normal-mode approach will lead in a simple way to a set of first-order differential equations.

In $\S 2$ we obtain the normal modes of the Alfven and sound waves within a linear analysis of the MHD equations. In $\S 3$ we derive a coupled set of equations for the three-wave interaction. In $\S 4$ we consider the case of a large-amplitude Alfvén wave acting as a pump to excite another Alfvén and a sound wave. It is shown that, according to the nature of the pump wave (standing or propagating), there can exist two distinct types of parametric instabilities (§§4.1 and 4.2). 


\section{The linear equations}

Let us consider the case of Alfvén and sound waves both propagating along an externally-imposed magnetic field, in a uniform, unbounded plasma. The equilibrium state of the plasma is characterized by a density $\rho_{0}$, a scalar pressure $p_{0}$, a zero drift velocity $\left(\mathrm{V}_{0}=0\right)$, and a steady magnetic field $\mathbf{H}_{0}=H_{0} \mathbf{z}$.

In an ideally conducting, compressible plasma, the Alfvén wave, as well as the acoustic wave, can be appropriately described by the MHD equations (Van Kampen \& Felderhof 1967)

$$
\begin{gathered}
\frac{\partial \rho}{\partial t}+\boldsymbol{\nabla} . \rho \mathbf{V}=0 \\
\rho \frac{\partial \mathbf{V}}{\partial t}+\rho(\mathbf{V} \cdot \boldsymbol{\nabla}) \mathbf{V}=-\nabla p-\frac{1}{4 \pi} \mathbf{H} \times(\boldsymbol{\nabla} \times \mathbf{H}), \\
\frac{\partial \mathbf{H}}{\partial t}=\boldsymbol{\nabla} \times(\mathbf{V} \times \mathbf{H}),
\end{gathered}
$$

together with an equation of state

$$
p=f(\rho) \text {. }
$$

To obtain some approximate solution of this highly nonlinear coupled system, we shall first study its normal modes within a linear analysis.

Let

$$
\mathbf{H}=\mathbf{H}_{\mathbf{0}}+\mathbf{H}_{\perp}, \quad \mathbf{V}=\mathbf{V}_{\|}+\mathbf{V}_{\perp}, \quad \rho=\rho_{\mathbf{0}}+\tilde{\rho},
$$

where the subscripts $\|$ and $\perp$ refer to the components parallel and perpendicular to the static magnetic field. The linearized form of (1) can be expressed as

$$
\begin{gathered}
\frac{\partial \tilde{\rho}}{\partial t}+\rho_{0} \frac{\partial V_{U}}{\partial z}=0, \\
\rho_{0} \frac{\partial V_{U}}{\partial t}+c_{S}^{2} \frac{\partial \tilde{\rho}}{\partial z}=0, \\
\frac{\partial H_{\perp}}{\partial t}-H_{0} \frac{\partial V_{\perp}}{\partial z}=0, \\
\frac{\partial V_{\perp}}{\partial t}-\frac{c_{A}^{2}}{H_{0}} \frac{\partial H_{\perp}}{\partial z}=0,
\end{gathered}
$$

where we define

$$
c_{S}^{2}=\left.\frac{d f(\rho)}{d \rho}\right|_{\rho=\rho_{0}} \quad \text { and } \quad c_{A}^{2}=\frac{H_{0}^{2}}{4 \pi \rho_{0}} .
$$

This system can easily be solved by introducing the normal modes of the Alfvén and sound waves, defined as

$$
a=\tilde{\rho}+c_{1} V_{\| \prime}, \quad b=H_{\perp}+c_{2} V_{\perp},
$$

with the coefficients $c_{1}$ and $c_{2}$ chosen such that

$$
\frac{\partial a}{\partial t}=-i \omega_{S} a, \quad \frac{\partial b}{\partial t}=-i \omega_{A} b
$$


$\omega_{S}$ and $\omega_{A}$ being the frequencies of the linear waves under consideration. By taking a linear combination of $(3 a, b)$, one immediately gets

$$
\frac{\partial a}{\partial t}+\rho_{0} \frac{\partial V_{\|}}{\partial z}+\frac{c_{1} c_{S}^{2}}{\rho_{0}} \frac{\partial \tilde{\rho}}{\partial z}=0
$$

Assuming a spatial dependence of the form $\exp \left\{i k_{S} z\right\}$, and using $(4 c)$ one then obtains the dispersion law

$$
\omega_{S}^{2}=c_{S}^{2} k_{S}^{2} \text { and } \quad c_{1}= \pm \rho_{0} k_{S} / \omega_{S}
$$

Thus, for a given wave vector $\mathbf{k}_{S}$, there exist two normal modes of the sound wave

and

$$
\begin{aligned}
& a^{+}=\tilde{\rho}^{+}+\frac{k_{S}}{\omega_{S}} \rho_{0} V_{\|^{+}} \sim \exp \left\{i k_{S} z-i \omega_{S} t\right\}, \\
& a^{-}=\tilde{\rho}^{-}-\frac{k_{S}}{\omega_{S}} \rho_{0} V_{\|}^{-} \sim \exp \left\{i k_{S} z+i \omega_{S} t\right\} .
\end{aligned}
$$

Clearly, $a^{+}$and $a^{-}$correspond to the forward and backward propagating waves with respect to the wave vector $\mathbf{k}_{S}$ (of course, $\omega_{S}$ is here defined as a real positive quantity).

In a quite analogous way, one obtains the normal modes of the Alfvén wave

$$
\begin{gathered}
b^{+}=H_{\perp}^{+}-\frac{k_{A}}{\omega_{A}} H_{0} V_{\perp}^{+} \sim \exp \left\{i k_{A} z-i \omega_{A} t\right\} \\
b^{-}=H_{\perp}^{-}+\frac{k_{A}}{\omega_{A}} H_{0} V_{\perp}^{-} \sim \exp \left\{i k_{A} z+i \omega_{A} t\right\}
\end{gathered}
$$

with the dispersion law

$$
\omega_{A}^{2}=c_{A}^{2} k_{A}^{2} \quad \text { and } \quad c_{2}=\mp H_{0} k_{A} / \omega_{A} .
$$

Before going on to a nonlinear analysis, let us express the perturbed quantities $\tilde{\rho}, V_{\|}, V_{\perp}$ and $H_{\perp}$ in terms of the normal modes $a$ and $b$. This is readily done by means of (3) and (6)-(8):

$$
\tilde{\rho}^{ \pm}=\frac{1}{2} a^{ \pm}, \quad V_{\mathbb{1}}^{ \pm}= \pm \frac{\omega_{S}}{2 \rho_{0} k_{S}} a^{ \pm}, \quad V_{\perp}^{ \pm}=\mp \frac{\omega_{A}}{2 H_{0} k_{A}} b^{ \pm}, \quad H_{\perp}^{ \pm}=\frac{1}{2} b^{ \pm} . \quad(9 a-d)
$$

\section{The coupled equations}

We now consider the interaction between a sound wave and two Alfvén waves (denoted by indices 1 and 2). Here nonlinear terms corresponding to the harmonic generation of these waves will be neglected. Equations $(1 a-d)$ then reduce to

$$
\begin{gathered}
\frac{\partial \tilde{\rho}}{\partial t}+\rho_{0} \frac{\partial V_{\|}}{\partial z}=0 \\
\frac{\partial V_{\|}}{\partial t}+\frac{c_{S}^{2}}{\rho_{0}} \frac{\partial \tilde{\rho}}{\partial z}=-\frac{1}{4 \pi \rho_{0}} \frac{\partial}{\partial z}\left(H_{1} \cdot H_{2}\right) \\
\frac{\partial H_{1}}{\partial t}-H_{0} \frac{\partial V_{1}}{\partial z}=-\frac{\partial}{\partial z}\left(V_{\|} \cdot H_{2}\right)
\end{gathered}
$$




$$
\begin{aligned}
& \frac{\partial V_{1}}{\partial t}-\frac{c_{A}^{2}}{H_{0}} \frac{\partial H_{1}}{\partial z}=-V_{\|} \cdot \frac{\partial V_{2}}{\partial z}-\frac{c_{A}^{2}}{\rho_{0} H_{0}} \tilde{\rho} \cdot \frac{\partial H_{2}}{\partial z}, \\
& \frac{\partial H_{2}}{\partial t}-H_{0} \frac{\partial V_{2}}{\partial z}=-\frac{\partial}{\partial z}\left(V_{n} \cdot H_{1}\right), \\
& \frac{\partial V_{2}}{\partial t}-\frac{c_{A}^{2}}{\bar{H}_{0}} \frac{\partial H_{2}}{\partial z}=-V_{\mathbb{1}} \cdot \frac{\partial V_{1}}{\partial z}-\frac{c_{A}^{2}}{\rho_{0} H_{0}} \tilde{\rho} \cdot \frac{\partial H_{1}}{\partial z},
\end{aligned}
$$

where the sign $\perp$ referring to the transverse components of the Alfvén waves, has been omitted.

In a low $\beta$ plasma $\left(c_{S}^{2} \ll c_{A}^{2}\right),(10 c-f)$ can be further simplified by neglecting the first terms on the right-hand sides, because they are small compared with the second terms.

Taking the same linear combination of $(10 a-f)$ as we did to obtain (5), we now have

$$
\begin{gathered}
\frac{\partial a^{ \pm}}{\partial t} \pm c_{S} \frac{\partial a^{ \pm}}{\partial z}=\mp \frac{k_{S}}{4 \pi \omega_{S}} \frac{\partial}{\partial z}\left(H_{1} \cdot H_{2}\right), \\
\frac{\partial b_{1}^{ \pm}}{\partial t} \pm c_{A} \frac{\partial b_{1}^{ \pm}}{\partial z}= \pm \frac{c_{A}^{2} k_{1}}{\rho_{0} \omega_{1}} \tilde{\rho} \cdot \frac{\partial H_{2}}{\partial z} \\
\frac{\partial b_{2}^{ \pm}}{\partial t} \pm c_{A} \frac{\partial b_{2}^{ \pm}}{\partial z}= \pm \frac{c_{A}^{2} k_{2}}{\rho_{0} \omega_{2}} \tilde{\rho} \cdot \frac{\partial H_{1}}{\partial z} .
\end{gathered}
$$

As such, this system is still intractable, except for the case of weak coupling. But one can look for solutions in the form of plane waves with 'slowly' varying amplitudes, i.e.

$$
\begin{aligned}
& a \pm=A \pm(t) \exp \left\{i k_{S} z \mp i \omega_{S} t\right\}+\text { complex conjugate, } \\
& b \pm=B \pm(t) \exp \left\{i k_{A} z \mp i \omega_{\Delta} t\right\}+\text { complex conjugate, }
\end{aligned}
$$

with

$$
\left|\frac{d}{d t} \ln A \pm\right|, \quad\left|\frac{d}{d t} \ln B \pm\right| \ll \omega_{A} .
$$

On substituting these solutions into $(11 a)$, and using (9) to express $\tilde{\rho}^{ \pm}, H_{1,2}^{ \pm}$in terms of $a^{ \pm}$and $b_{1,2}^{ \pm}$, we readily obtain $\dagger$

$$
\begin{aligned}
\left(\omega_{S}, k_{S}\right) \frac{\partial A^{+}}{\partial t}+( & \left.-\omega_{S},-k_{S}\right) \frac{\partial \bar{A}^{+}}{\partial t} \\
= & \frac{-k_{S}}{16 \pi \omega_{S}} \frac{\partial}{\partial z}\left\{\left[B_{1}^{+} B_{2}^{+}\left(\omega_{1}+\omega_{2}, k_{1}+k_{2}\right)+B_{1}^{+} B_{2}^{-}\left(\omega_{1}-\omega_{2}, k_{1}+k_{2}\right)\right.\right. \\
& +B_{1}^{-} B_{2}^{+}\left(-\omega_{1}+\omega_{2}, k_{1}+k_{2}\right)+B_{1}^{-} B_{2}^{-}\left(-\omega_{1}-\omega_{2}, k_{1}+k_{2}\right) \\
& +B_{1}^{+} \bar{B}_{2}^{+}\left(\omega_{1}-\omega_{2}, k_{1}-k_{2}\right)+B_{1}^{+} \bar{B}_{2}^{-}\left(\omega_{1}+\omega_{2}, k_{1}-k_{2}\right) \\
& \left.+B_{1}^{-} \bar{B}_{2}^{+}\left(-\omega_{1}-\omega_{2}, k_{1}-k_{2}\right)+B_{1}^{-} \bar{B}_{2}^{-}\left(-\omega_{1}+\omega_{2}, k_{1}-k_{2}\right)\right] \\
& + \text { complex conjugate }\}
\end{aligned}
$$

where a barred quantity stands for its complex conjugate, and the abbreviation $(\omega, k)$ stands for $\exp \{i k z-i \omega t\}$.

$\dagger$ In calculating the coupling terms on the right-hand side of (11), one must allow for the influence of both the forward and backward modes. 
Let us now consider only the interaction between three waves satisfying the conditions

$$
\mathbf{k}_{1}-\mathbf{k}_{2}=\mathbf{k}_{S}, \quad \omega_{1}-\omega_{2}=\delta \approx \omega_{S} .
$$

On substituting (14) into (13), and keeping only terms with approximately the same oscillating exponentials on both sides of (13), this will reduce to

$$
\begin{aligned}
& \frac{\partial A^{+}}{\partial t}+\left(-2 \omega_{S},-2 k_{S}\right) \frac{\partial \bar{A}^{+}}{\partial t} \\
& =\frac{-i k_{S}^{2}}{16 \pi \omega_{S}}\left[B_{1}^{+} \bar{B}_{2}^{+}\left(\delta-\omega_{S}, 0\right)+B_{1}^{-} \bar{B}_{2}^{-}\left(-\delta-\omega_{S}, 0\right)\right. \\
& \left.\quad-\bar{B}_{1}^{+} B_{2}^{+}\left(-\delta-\omega_{S},-2 k_{S}\right)-\bar{B}_{1}^{-} B_{2}^{-}\left(\delta-\omega_{S},-2 k_{S}\right)\right]
\end{aligned}
$$

Noting that the mode amplitudes are only time-dependent, we can therefore take a space average of (15) and obtain

$$
\frac{\partial A^{+}}{\partial t}=\frac{-i k_{S}^{2}}{16 \pi \omega_{S}}\left[B_{1}^{+} \bar{B}_{2}^{+} \exp \left\{-i\left(\delta-\omega_{S}\right) t\right\}+B_{1}^{-} \bar{B}_{2}^{-} \exp \left\{i\left(\delta+\omega_{S}\right) t\right\}\right] .
$$

In a quite similar way, one gets

$$
\begin{aligned}
& \frac{\partial A^{-}}{\partial t}=\frac{i k_{S}^{2}}{16 \pi \omega_{S}}\left[B_{1}^{+} \bar{B}_{2}^{+} \exp \left\{-i\left(\delta+\omega_{S}\right) t\right\}+B_{1}^{-} \bar{B}_{2}^{-} \exp \left\{i\left(\delta-\omega_{S}\right) t\right\}\right], \\
& \frac{\partial B_{1}^{ \pm}}{\partial t}=\frac{ \pm i k_{1} k_{2} c_{A}^{2}}{4 \rho_{0} \omega_{1}}\left[A^{+} B_{2}^{ \pm} \exp \left\{ \pm i\left(\delta \mp \omega_{S}\right) t\right\}+A-B_{2}^{ \pm} \exp \left\{ \pm i\left(\delta \pm \omega_{S}\right) t\right\}\right] \\
& \frac{\partial B_{2}^{ \pm}}{\partial t}=\frac{ \pm i k_{1} k_{2} c_{A}^{2}}{4 \rho_{0} \omega_{2}}\left[\bar{A}^{+} B_{1}^{ \pm} \exp \left\{\mp i\left(\delta \mp \omega_{S}\right) t\right\}+\bar{A}^{-} B_{1}^{ \pm} \exp \left\{\mp i\left(\delta \pm \omega_{S}\right) t\right\}\right]
\end{aligned}
$$

Equations $(16 a-d)$ represent the nonlinear coupling between 6 normal modes treated on the same basis. In $\S 4$ we shall consider the case of parametric coupling in which one of the waves has an amplitude much larger than that of the others, and can be treated as constant.

\section{The linearized coupled equations}

In various situations, one deals with a strong, externally-imposed electromagnetic field in a plasma. The field will change the behaviour of the plasma with respect to small disturbances. The problem then is to know how these disturbances will develop in the medium, and whether they can be driven unstable.

Here we consider the case of a large amplitude Alfvén wave $b_{1}$, acting as a pump of constant power. Using the coupled equations derived in $\$ 3$, we shall determine the conditions under which some initially small perturbation grows in the form of an Alfvén and a sound wave. Within this approximation (constant pump power), (16) reduces to a simple set of linear equations describing the parametric coupling of $A \pm$ and $B_{2}^{ \pm}$:

$$
\frac{\partial A^{ \pm}}{\partial t}=\frac{\mp i k_{S}^{2}}{16 \pi \omega_{S}}\left[B_{1}^{+} \bar{B}_{2}^{+} \exp \left\{-i\left(\delta \mp \omega_{S}\right) t\right\}+B_{1}^{-} \bar{B}_{2}^{-} \exp \left\{i\left(\delta \pm \omega_{S}\right) t\right\}\right]
$$




$$
\begin{aligned}
\frac{\partial B_{2}^{ \pm}}{\partial t} & =\frac{ \pm i k_{1} k_{2} c_{A}^{2}}{4 \rho_{0} \omega_{2}}\left[B_{1}^{ \pm} \bar{A}^{+} \exp \left\{\mp i\left(\delta \mp \omega_{S}\right) t\right\}+B_{1}^{ \pm} \bar{A}^{-} \exp \left\{\mp i\left(\delta \pm \omega_{S}\right) t\right\}\right], \\
B_{1}^{ \pm} & =\text {const. }
\end{aligned}
$$

Equations $(17 a-c)$ can readily be solved using the transformation

$$
\left.\begin{array}{c}
X^{+}=A^{+}, \quad X^{-}=A^{-} \exp \left\{2 i \omega_{S} t\right\}, \\
Y^{+}=\bar{B}_{2}^{+} \exp \left\{-i\left(\delta-\omega_{S}\right) t\right\}, \quad Y^{-}=\bar{B}_{2}^{-} \exp \left\{i\left(\delta+\omega_{S}\right) t\right\} .
\end{array}\right\}
$$

On substituting these new variables in (17), and assuming solutions of the form $\exp \{-i \omega t\}$, we obtain

$$
\left[\begin{array}{cccc}
\omega & 0 & -\frac{k_{S}^{2} B_{1}^{+}}{16 \pi \omega_{S}} & -\frac{k_{S}^{2} B_{1}^{-}}{16 \pi \omega_{S}} \\
0 & \omega+2 \omega_{S} & \frac{k_{S}^{2} B_{1}^{+}}{16 \pi \omega_{S}} & \frac{k_{S}^{2} B_{1}^{-}}{16 \pi \omega_{S}} \\
-\frac{k_{1} k_{2} c_{A}^{2} \bar{B}_{1}^{+}}{4 \rho_{0} \omega_{2}} & -\frac{k_{1} k_{2} c_{A}^{2} \bar{B}_{1}^{+}}{4 \rho_{0} \omega_{2}} & \omega+\omega_{S}-\delta & 0 \\
\frac{k_{1} k_{2} c_{A}^{2} \bar{B}_{1}^{-}}{4 \rho_{0} \omega_{2}} & \frac{k_{1} k_{2} c_{A}^{2} \bar{B}_{1}^{-}}{4 \rho_{0} \omega_{2}} & 0 & \omega+\omega_{S}+\delta
\end{array}\right]\left[\begin{array}{l}
X^{+} \\
X^{-} \\
Y^{+} \\
Y^{-}
\end{array}\right]=0
$$

which yields the dispersion law

$$
\begin{aligned}
\omega\left(\omega+2 \omega_{S}\right)\left(\omega+\omega_{S}+\delta\right) & \left(\omega+\omega_{S}-\delta\right) \\
= & \frac{k_{1} k_{2} k_{S}^{2} c_{A}^{2}}{32 \pi \rho_{0} \omega_{2}}\left[\left|B_{1}^{+}\right|^{2}\left(\omega+\omega_{S}+\delta\right)-\left|B_{1}^{-}\right|^{2}\left(\omega+\omega_{S}-\delta\right)\right] .
\end{aligned}
$$

A first view of this equation suggests that one consider separately the two cases of the standing and the propagating pump.

\subsection{Standing pump}

In this case, we have

$$
\left|B_{1}^{+}\right|=\left|B_{1}^{-}\right|
$$

and (20) takes the form

$$
\left(\Omega_{S}^{2}-\omega_{S}^{2}\right)\left(\Omega_{S}^{2}-\delta^{2}\right)-K \delta=0
$$

where

$$
\Omega_{S}=\omega+\omega_{S}, \quad K=\frac{k_{1} k_{2} k_{S}^{2} c_{A}^{2}\left|B_{1}^{+}\right|^{2}}{16 \pi \rho_{0} \omega_{2}}
$$

if we separate the real and imaginary parts of $\Omega_{S}$, and write

$$
\Omega_{S}=\Omega_{r}+i \gamma
$$

then $\Omega_{r}$ and $\gamma$ represent the frequency and growth $(\gamma>0)$ or damping $(\gamma<0)$ rate of the sound wave, respectively. Physically, one should distinguish the two cases: (i) $\Omega_{r} \neq 0, \gamma>0$, corresponding to an oscillating instability; and (ii) $\Omega_{r}=0, \gamma>0$, corresponding to a purely growing instability. From its structure, it is easily shown that $(22 a)$ can admit either purely growing or oscillating solutions, depending on the sign of $K \delta$. 
4.1.1. $K \delta>0$. In this case, there can only exist purely growing solutions given by

$$
\Omega_{r}=0, \quad \gamma^{2}=\frac{1}{2}\left\{\left[\left(\omega_{S}^{2}-\delta^{2}\right)^{2}+4 K \delta\right]^{\frac{1}{2}}-\left(\omega_{S}^{2}+\delta^{2}\right)\right\} .
$$

The threshold pump intensity for excitation of this wave is

$$
K_{m}=\omega_{S}^{2} \delta
$$

or, in terms of the pump field $H_{1}^{+}$,

$$
\frac{\left|H_{1}^{+}\right|_{m}^{2}}{4 \pi \rho_{0}}=\left|\frac{\delta}{k_{1}}\right|\left(\frac{c_{S}}{c_{A}}\right)^{2} c_{A^{4}} .
$$

For a given pump intensity, the maximum growth rate is attained at

$$
\delta_{M}=\left(\frac{K}{4}+\left[\frac{K^{2}}{16}+\frac{\omega_{S}^{6}}{27}\right]^{\frac{1}{2}}\right)^{\frac{1}{3}}+\left(\frac{K}{4}-\left[\frac{K^{2}}{16}+\frac{\omega_{S}^{6}}{27}\right]^{\frac{1}{2}}\right)^{\frac{1}{3}},
$$

which reduces to

$$
\delta_{M}=\left(\frac{1}{2} K\right)^{\frac{1}{3}}
$$

in the case of a 'strong' pump $\left(|K| \gg \omega_{S}^{3}\right)$. For a 'weak' pump $\left(|K| \ll \omega_{S}^{3}\right)$ one has

$$
\delta_{M}=K /\left(2 \omega_{S}^{2}\right) \text {. }
$$

The maximum growth rates are given by (strong pump)

$$
\begin{aligned}
& \gamma_{M I}=\left(\frac{1}{2}|K|\right)^{\frac{1}{3}}, \\
& \gamma_{M}=|K| /\left(2 \omega_{S}^{2}\right) .
\end{aligned}
$$

From these equations, it is noticeable that the maximum growth rate increases much more sensitively with the pump power in the weak pump regime than it does for strong pumps.

4.1.2. $K \delta<0$. As not in the previous case, $(22 a)$ now has only oscillating solutions. The frequency and growth rate of the sound wave are given by

and

$$
\begin{gathered}
\Omega_{r}^{2}=\gamma^{2}+\frac{1}{2}\left(\omega_{S}^{2}+\delta^{2}\right), \\
\gamma^{2}=-\frac{1}{4}\left(\omega_{S}^{2}+\delta^{2}\right)+\frac{1}{2}\left(\omega_{S}^{2} \delta^{2}-K \delta\right)^{\frac{1}{2}} .
\end{gathered}
$$

The threshold power for excitation of this wave is

$$
K_{m}=-\left(\omega_{\mathrm{S}}^{2}-\delta^{2}\right)^{2} /(4 \delta) .
$$

In the weak pump regime, the growth rate attains its maximum value

$$
\gamma_{M}=\left[|K| /\left(4 \omega_{S}\right)\right]^{\frac{1}{2}}
$$

at the perfect matching frequency

$$
\delta_{M}=\omega_{S}
$$

Then, the frequency of the sound wave becomes

$$
\Omega_{r}=\left(1+\frac{|K|}{4 \omega_{S}^{3}}\right)^{\frac{1}{2}} \omega_{S}
$$


For a strong pump, one obtains

$$
\gamma_{M}=\frac{\sqrt{ } 3}{2}\left(\frac{|K|}{4}\right)^{\frac{1}{3}}, \quad \delta_{M}=-\left(\frac{K}{4}\right)^{\frac{1}{3}} \quad \text { and } \quad \Omega_{r}=\frac{\sqrt{ } 5}{2}\left(\frac{|K|}{4}\right)^{\frac{1}{3}} .
$$

In both cases ( $K \delta>0$ and $K \delta<0), K$ is allowed to be either positive or negative corresponding to the forward or backward scattering of the Alfvén wave, respectively.

This is the case where

\subsection{Propagating pump}

$$
B_{1}^{-}=0 .
$$

From $(17 b)$ and (20), one sees that the backward mode $b_{2}^{-}$is not excited, while the three modes $a^{+}, a^{-}$and $b_{2}^{+}$are coupled according to the dispersion relation

or

$$
\begin{aligned}
\omega\left(\omega+2 \omega_{S}\right)\left(\omega+\omega_{S}-\delta\right)-\frac{1}{2} K & =0, \\
\Omega_{S}^{3}-\delta \Omega_{S}^{2}-\omega_{S}^{2} \Omega_{S}+\delta \omega_{S}^{2}-\frac{1}{2} K & =0,
\end{aligned}
$$

which can admit unstable solutions if the pump intensity exceeds the threshold value

$$
K_{m}=\frac{4}{27}\left[-\delta^{3}+9 \delta \omega_{S}^{2} \mp\left(3 \omega_{S}^{2}+\delta^{2}\right)^{\frac{3}{2}}\right] .
$$

For $|K|>\left|K_{m}\right|$, the frequency and growth rate of the (unstable) sound wave are given by

where

$$
\Omega_{r}=\frac{\delta}{3}+\frac{x+y}{2}, \quad \gamma=\frac{\sqrt{ } 3}{2}|x-y|,
$$

$$
\left(\begin{array}{l}
x \\
y
\end{array}\right)=\frac{1}{3}\left\{\left(-\delta^{3}+9 \delta \omega_{S}^{2}-\frac{27}{4} K\right) \pm\left[\left(-\delta^{3}+9 \delta \omega_{S}^{2}-\frac{27}{4} K\right)^{2}-\left(3 \omega_{S}^{2}+\delta^{2}\right)^{3}\right]^{\frac{1}{2}}\right\}^{\frac{1}{3}}
$$

In the low threshold region, (35) yields the values

and

$$
\Omega_{r}=\frac{\delta}{3}+\frac{\left(9 \delta \omega_{S}^{2}-\delta^{3}\right)^{\frac{1}{3}}}{3}\left(1-\frac{9 K}{4\left(9 \delta \omega_{S}^{2}-\delta^{3}\right)}\right),
$$

Putting $\delta=\omega_{S}$ in (36), one obtains

$$
\Omega_{r}=\omega_{S}-\frac{3 K}{16 \omega_{S}^{2}} \quad \text { and } \quad \gamma=\frac{1}{2}\left(-\frac{K}{\omega_{S}}\right)^{\frac{1}{2}} .
$$

This can be compared with the results of Sagdeev \& Galeev (1969) in the same situation (i.e. propagating pump under perfect matching conditions), written in our notation

$$
\Omega_{r}=\omega_{S}, \quad \gamma=\left(-K / \omega_{S}\right)^{\frac{1}{2}}
$$

where one notices that the frequency shift is zero, and the growth rate is twice that given by $(37 b)$. While the first discrepancy stems from the space and time averaging process used in their theory, the second is due to the fact that they have 
not accounted for the 'slow-time' dependence of the fluid density in the mass conservation equation. Also their theory, using the space and time averaging, does not allow for any frequency mismatch; neither does it allow for any influence of the backward sound wave.

\section{Conclusion}

From the foregoing analysis, it appears that (i) a standing pump can excite both oscillating and non-oscillating sound waves ; (ii) a propagating pump can excite only propagating waves; (iii) owing to its low frequency (compared with the Alfven wave), the backward sound wave can couple efficiently with the Alfven waves; (iv) the growth rates of the excited waves depend on the pump intensity in a characteristic way $\left(K, K^{\frac{1}{2}}\right.$ or $\left.K^{\frac{1}{3}}\right)$, according to the pump regime (weak or strong). Finally, in $\$ 4$ we assumed that the pump amplitude remains constant during the coupling process. Although this is a very good approximation in the case of parametric excitation, it can be easily avoided. In fact, solutions to the nonlinear system (16) can be expressed exactly in terms of elliptic integrals (Amstrong et al. 1962).

The author thanks Dr F Hofmann and Professor E.S. Weibel for reading the manuscript and commenting on it. This work was performed under the auspices of the Fonds National Suisse de la Recherche Scientifique.

\section{REFERENCES}

Amstrona, J. A., Bloembergen, N., Ducuing, J. \& Persham, P. S. 1962 Phys. Rev. 127, 1918.

DuBoIs, D.F. 1972 Interaction of intense radiation with plasmas. Lectures Given at the Department of Astro-Geophysics, University of Colorado.

Kadomtsev, B. B. 1965 Plasma Turbulence. Academic.

Sagdeev, R. Z. \& Galeev, A. A. 1969 Nonlinear Plasma Theory. Benjamin.

Van Kanpen, N. G. \& Felderhof, B.U. 1967 Theoretical Methods in Plasma Physics. North-Holland. 